\title{
Kinematic Analysis of Instruments for Minimal- ly Invasive Robotic Surgery: Generalization of the Reference Task
}

\author{
B. Deutschmann, R. Konietschke and C. Ott \\ Institute of Robotics and Mechatronics, German Aerospace Center (DLR) \\ bastian.deutschmann@dlr.de
}

\begin{abstract}
In minimally invasive robotic surgery, actuated instruments are used that provide additional degrees of freedom (DoF) inside the human body. Kinematic limitations due to the instrument could endanger the secure execution of a surgical task. Numerous design alternatives are proposed in the literature whereas little work is done that evaluates the performance of these instruments in an objective way. This paper presents recent extensions towards a method from [1] to evaluate alternative designs of instrument kinematics with respect to their ability to perform surgical tasks. These extensions include further analysis of the task "suturing", one of the key tasks in robotic surgery, to extract the main components and generalize it with respect to the arbitrariness in which way this task occurs during a minimally invasive intervention. The paper concludes with more recent evaluation results and gives recommendations for instruments and their kinematic structure.
\end{abstract}

Key words: Medical robotics, robotic telesurgery, kinematics, workspace analysis, laparoscopic suturing

\section{Introduction}

In robot guided minimally invasive interventions, the possibility of in situ manipulation without workspace limitations and a secure execution of the surgical task are amongst the main demands of the surgeons. In this context intracorporeal suturing is considered as the fundamental task [2]. Commonly, the robotic system on the outside of the patient is responsible for the positioning of the tool center point (TCP). In contrast, the robotized instrument with its wrist like joints at the tip, acting inside the patient, is mainly responsible for the orientation of the TCP. Suturing requires a great amount of maneuverability and flexibility from the instrument wrist-like joints [3]. Limitations of this wrist kinematics would endanger the severe procedure of placing a suture. 
Numerous design proposals of instrument kinematics for minimally invasive surgery can be found in the literature [4]-[8]. A performance evaluation of these proposals regarding surgical tasks is not stated. However, a method that incorporates an evaluation of performance based on a relevant task would be exceptionally useful prior to building prototypes to reduce the cost and save time. Furthermore, the importance of kinematic design parameters, e.g. the joint sequence or the range of motion of a joint, can be identified to guide mechanical designers.

In minimally invasive surgery, the relative position of the entry port with respect to the area of interest, i.e. the setup, is patient specific. However, this setup greatly influences the performance of an instrument regarding a desired task, e.g. placing a suture. Therefore, an evaluation method that is independent of this setup would be of main concern.

Related publications are for example the work of Çavuşoğlu et al. [9], which evaluated two instrument kinematics with respect to their ability to perform a surgical knot. Or Sallé et al. [10] which evaluated arbitrary axis arrangements for a redundant instrument to be able to perform a complete anastomosis for coronary artery bypass. Both methods incorporate quantitative criteria to account for e.g. the manipulability while performing the task. For a detailed state of the art see [1].

According to [11], a method that evaluates the performance of a manipulator needs to clearly specify the task and the tool separately in order to determine the respective influence on the overall performance. The task specific evaluation method published in [1] incorporates this clear specification and is used as a basis for the current work. The focus of the paper at hand is the development of a more generalized task description by

1. a more detailed examination of the suturing task including a classification into the two subtasks of "stitching" and "knot tying"

2. a uniform sampling method for the rotation group $S O(3)$ to characterize the task probabilistically [11].

The generalization provides a task that includes motions mainly affecting the instruments capabilities which contributes to a precise evaluation process. The aforementioned feature and the method's independence on the setup is the main benefit and uniqueness of the method compared with other evaluation methods [9], [10]. The paper concludes with recommendations for the kinematic designs of instruments with respect to the task of suturing.

The paper is structured by four sections. In Sec. 2, the proposed method will be surveyed whereas Sec. 3 assesses the key component, the task description, in a more detailed way. Sec. 4 presents three examples and Sec. 5 revises benefits and limitations of the method and gives perspectives for future developments. 


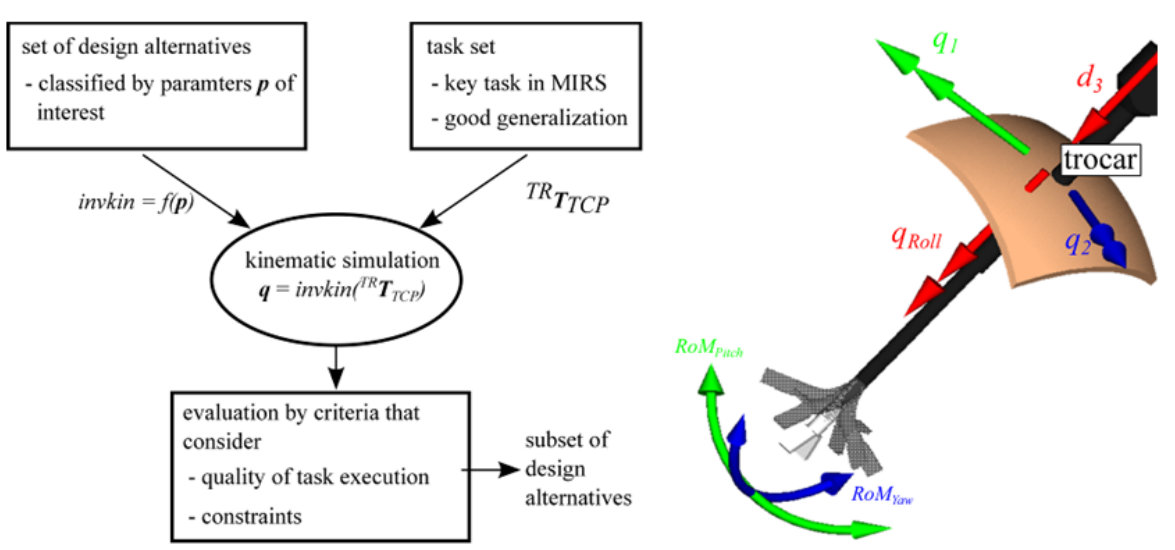

Fig 1: Left: Course of the evaluation method. Right: Abstraction of a minimally invasive instrument with trocar and six resulting DoF's (Image adopted from [14]).

\section{Method}

The proposed method aims at evaluating the performance of kinematic design alternatives of instruments for minimally invasive surgery with respect to a given task, prior to building them. The course of the method is presented in Fig 1, left. Following [11], the "tool" and the "task" need to be clearly specified as a primary step. Here, "tool" is referred to as "set of design alternatives". Subsequently, kinematic simulations are performed yielding the joint angles of the instrument, necessary to execute the task. Based on these joint angles, the predefined success ratio "SUR" and Shaft Distance " $d_{I B}$ " criteria are applied to evaluate the performance. The "SUR" value accounts for the amount of successfully performed tasks and it ranges from 0 to $100 \%$. The " $d_{I B}$ " value accounts for the consumed space during task execution and is only bounded from below. High values for "SUR" and low values for " $d_{I B}$ " are desirable. Except for the task set presented in Sec.3, the mentioned parts of the method are already published in [1]. However, parameters used to characterize the instruments and to configure the simulation are briefly recalled in the following.

An instrument is considered as a manipulator with $6 \mathrm{DoF}$ attached to the trocar i.e. the entry point to the patient, see Fig 1 right. Here, the reference frame $\boldsymbol{T}_{T r}$ for all other assigned frames is located, with $\mathrm{x}$-axis pointing along the shaft of the instrument. At the trocar, $3 \mathrm{DoF}$ are located with joint angles $q_{1}, q_{2}, d_{3}$, see Fig 1 right. These joint angles are independent of the instrument that is attached to it and are introduced to abstract the robot outside the patient. The instrument itself possesses $3 \mathrm{DoF}$ which are realized by a roll axis along the shaft, $q_{\text {Roll }}$, followed by an arbitrary sequence of pitch and yaw axes, $R o M_{\text {Pitch }}$ and $R o M_{\text {Yaw }}$. To omit redundancy, a linear relationship between subsequent pitch and subsequent yaw is im- 
plemented. Each instrument possesses a tool center point TCP ${ }^{\mathrm{Tr}} \boldsymbol{T}_{T C P}$, i.e. a frame at which for example a needle is grasped. Parameters, which are used to characterize instruments within the present method, are

1. The range of motion of the instrument: $R o M= \pm\left[q_{\text {Roll, lim }}, q_{\text {Pitch,lim, },}, q_{\text {Yaw,lim, },}\right]$

2. The total number of pitch and yaw joints $\left[\mathrm{N}_{\text {Pitch }}, \mathrm{N}_{\text {Yaw }}\right]$

3. The joint sequence, i.e. the order of the pitch and yaw joints

4. The total kinematic length $l_{k i n}$ i.e. the distance from the intersection of the first two axes (roll $\cap$ pitch/yaw), ending at the TCP.

Another important parameter is the initial length of the shaft, i.e. the initial distance of the trocar towards the instruments TCP frame ${ }^{\mathrm{Tr}} \boldsymbol{T}_{\mathrm{TCP}}$, denoted as ${ }^{{ }_{\mathrm{Tr}}} \boldsymbol{t}_{\mathrm{TCP}}$. For more details, see [1].

\section{Generalization of Tasks}

As stated by Sheridan [11], the choice and specification of the tool and the task are considered to be the crucial points in every evaluation method. Since the "tools", i.e. the instruments, are already specified, a generalized specification of the task is presented in the following. According to [11], the description of a task utilized for performance evaluation should contain 1) a probabilistic characterization of the initial and final configuration pose of the manipulator and 2) the description at all possible task trajectories, i.e. the steps in between initial and final configuration. With respect to these two points, the overall task of intracorporeal suturing will be specified as

1. An initial pose of the needle with respect to a pose of the incision line

2. An ascending "stitching" and "knot tying" trajectory including the final pose, after the knot is tight.

In the following paragraphs, these specifications are explained in more detail.

The acquisition of the intracorporeal suturing trajectory was done with a camera system, which tracked the TCP pose of a needle holder $(\mathrm{NH})$ while performing intracorporeal suture in an open surgery test bed. Five expert surgeons and one trainee were asked to do this. For details, see [1]. This acquisition yielded a trajectory of the TCP of the NH with respect to the cameras reference frame. For the further developments of the task, it is necessary to represent this trajectory with respect to the pose of the incision line (IL), yielding

$$
{ }^{I L} \boldsymbol{T}_{T C P}\left(t_{n}\right)=\left({ }^{R E F} \boldsymbol{T}_{I L}\right)^{-1} \cdot{ }^{R E F} \boldsymbol{T}_{T C P}\left(t_{n}\right)
$$



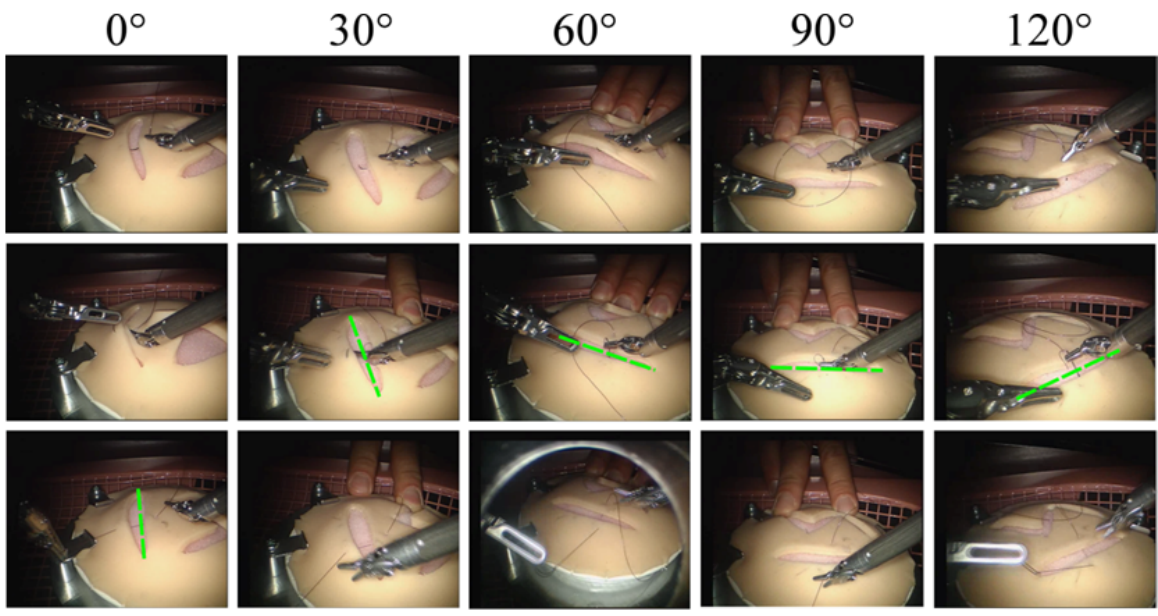

Fig 2: Orientation of the needle holder / needle during the subtask "stitching" of the task suturing w.r.t the orientation of the incision line (green). Images are shown exemplarily from one surgeon.

${ }^{R E F} \boldsymbol{T}_{I L}$ represents the pose of the IL calibrated before the tracking experiment. To further specify the task "intracorporeal suturing", this trajectory will be decomposed into two parts which are dependent and independent of location of the IL, see Section 3.1. To achieve the suggested probabilistic characterization of the task, a uniform sampling of the pose of IL is done which is explained in Section 3.2.

\subsection{Task decomposition}

According to [12], intracorporeal suturing can be decomposed into two major subtasks, which are "stitching" and "knot tying".

"Stitching" is the motion of the needle through the two flaps of the incision and consists of seven consecutive steps [12]: 1. Positioning the needle - 2. Grasping the tissue - 3. Pulling the needle through - 4. Re-positioning the needle - 5. Regrasping the tissue -6 . Re-pulling the needle through -7 . Pulling suture through. Obviously, this subtask can be considered as a close interaction with the incision line (IL) and surrounding tissue. Therefore, a first hypothesis is set up which is substantiated afterwards.

Hypothesis 1: "stitching" is dependent on the pose of the IL.

For the task "stitching", several guidelines exist that can be found in basic surgical skill literature [13]. First of all, a complete suture should provide a level surface with a symmetric, small amount of eversion of the edges. The sides of the wound should be well aligned, so that redundant tissue does not develop at the end of the IL and cause vertical or horizontal misalignment. Consequently, the virtual con- 



$120^{\circ}$
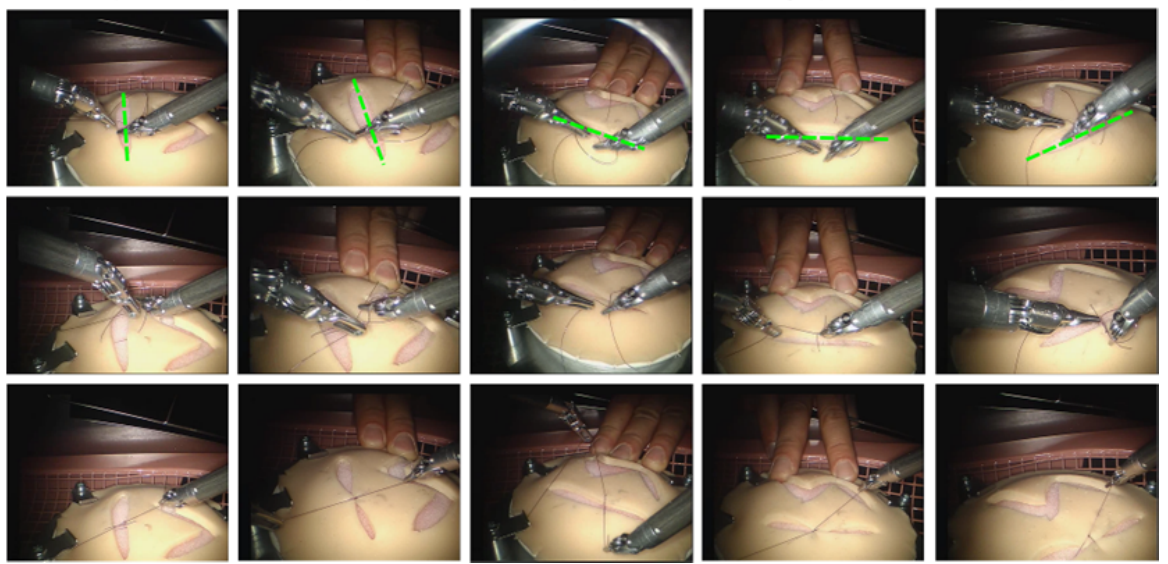

Fig 3: Orientation of the $\mathrm{NH} /$ needle during the subtask "knot tying" w.r.t. the orientation of the incision line (green). Images are shown exemplarily from one surgeon.

nection between the penetration into and out of the tissue should be perpendicular to the line of incision.

To further investigate this dependency of the "stitching" task, we performed an experiment with the daVinci( ) surgical system at the department of urology, university of Leipzig where 4 experienced surgeons were asked to perform an intracorporeal suture on a standardized test-bed [14]. The IL was rotated in steps of $30^{\circ}$, starting from $0^{\circ}$ and ending at $120^{\circ}$, to investigate whether the stitching motion adapts to the changed line of incision.

In Fig 2, motion details of a "stitching" motion with respect to 5 different orientation of the IL (green) are presented as image sequence of 3 images in 5 columns. First of all, it can be confirmed, that the assumption of the right angularity of the stitching motion with respect to the IL is correct. Needle (first and second row) and suture string (third row) can be observed to be perpendicular throughout every investigated angle of the IL. Furthermore, the axis along the branches of the needle holder is by observation parallel during the stitching of both wound sides. Both observations confirm hypothesis 1 .

The second subtask, "knot tying", is the motion of forming a loop and tightening the knot and consists of four consecutive steps [12]: 1. Positioning the needle and suture - 2. Forming loops - 3. Pulling short tail through loops - 4. Pulling knot tight. The steps $1-3$ are considered here to be free movements since there is no interaction with the line of incision and surrounding tissue. They are performed based on the surgeons experience and are adjusted to existing constraints in the workspace. Therefore, the following, second hypothesis is set up and substantiated afterwards.

Hypothesis 2: "knot tying" is independent on the orientation of the IL. 
Forming a proper knot in surgery is considered as a crucial and complex task, however the pure motion necessary to form the loops and tighten the knot is considered to require less then $6 \mathrm{DoF}$ but at least $3 \mathrm{DoF}$ [15]. Except from step 4 "pull the knot tight", the motion is not in direct contact with the tissue and therefore the space in which the motion takes place can be chosen freely within the confined space of a minimally invasive intervention. For this, hypothesis 2 holds. To substantiate this, again observations from the experiments with the daVinci@ surgical system at the department of urology, university of Leipzig are presented. In Fig 3 , motion details of a "knot tying" motion with respect to 5 different orientations of the IL ( 5 columns) are presented as an image sequence of 3 images. Although images of the respective steps vary, no correlation between the orientation of the IL and the orientation of the needle holder can be observed.

As a summary of the paragraph, intracorporeal suturing can be decomposed in two subtasks, the "stitching" motion, which is rather complex and dependent on the orientation of the IL, and the subtask "knot tying", a motion which is performed without having contact to the tissue and therefore needs less DoF. Moreover, it is independent on the orientation of the IL.

\subsection{Sampling of the line of incision}

An instrument has to enable the task intracorporeal suturing in a diversity of orientations in a minimally invasive intervention. To account for this diversity, the initial orientation of the $\mathrm{IL}^{T r} \boldsymbol{R}_{I L \text {,init }} \in S O(3)$ is sampled uniformly by using a method proposed in [16]. This method ensures a deterministic equivolumetric sampling of $S O(3)$, the space of 3 dimensional rotations. It is based on a multiresolution grid on sequences of Cartesian products, e.g. the Cartesian product of $\mathrm{S}^{1}$ and $\mathrm{S}^{2}$ creating the space $S O(3)$. The resulting grid of the space $S^{1} \otimes S^{2}$ has $N_{p}$ points, [16]. To generate the aforementioned uniform deterministic sequence over $S O(3)$, the open source $\mathrm{C}++$ library by Jain [17] is used. The output grid is parameterized using unit quaternions $(x, y, z, w)$. Thus, the initial orientation of the IL at each grid point $i$ is rotated

$$
\begin{aligned}
{ }^{T r} \boldsymbol{R}_{I L, i} & ={ }^{T r} \boldsymbol{R}_{I L, \text { init }}{ }^{I L, \text { init }} \boldsymbol{R}\left(x_{i}, y_{i}, z_{i}, w_{i}\right) \quad i=1 \cdots N_{p} \\
{ }^{T r} \boldsymbol{R}_{I L, i} & =\left[\begin{array}{cc}
{ }^{T r} \boldsymbol{R}_{I L, i} & { }^{T r} \boldsymbol{t}_{I L} \\
\underline{0}^{T} & 1
\end{array}\right]
\end{aligned}
$$

whereas ${ }^{\operatorname{Tr}} \boldsymbol{R}_{i}\left(x_{i}, y_{i}, z_{i}, w_{i}\right) \in S O(3)$ is one grid point of the sample set in $S O(3)$ and ${ }^{T r} \boldsymbol{t}_{I L}$ is the initial distance vector, starting at the global reference frame at the trocar and ending at the initial suture line, see Section 2. The creation of this uniform grid of initial IL orientation is illustrated in Fig 4. 


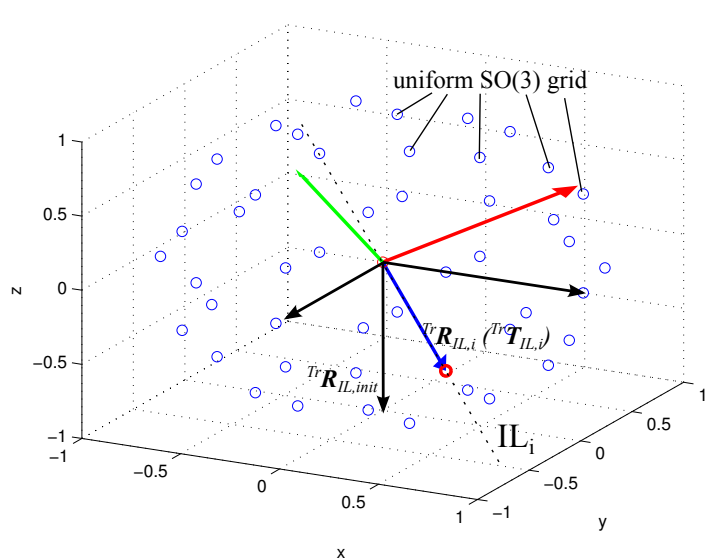

Fig 4: Creation uniform grid of ILs. The orientation of the initial incision line ${ }^{T r} \boldsymbol{R}_{I L \text {,init }}$ (black) is sampled with a the uniform grid of the $S O(3)$ of size $N_{p}$ created around the origin of ${ }^{T r} \boldsymbol{R}_{L, \text { ini }}$. A resulting incision line $I L_{i}$ with frame ${ }^{T r} \boldsymbol{R}_{L, i}$ (colored) is illustrated as an example.

\subsection{Overall Task - Summary}

The overall task for the proposed evaluation method is presented in a general manner, the initial pose is characterized probabilistically and the trajectory embodies a key task for the surgical field generalized for the evaluation of instruments. The task constitutes of the following elements. A suturing trajectory ${ }^{{ }^{L}} \boldsymbol{T}_{T C P}$ $\in R^{4 \times 4}$, see Equation (1), is performed at $N_{p}$ different initial orientation of the IL ${ }^{T r} \boldsymbol{T}_{I L, i} \in R^{4 \times 4}$, see Equation (2) and Fig 4 . The overall trajectory for the $\mathrm{i}^{\text {th }}$ grid point is described as

$$
{ }^{T r} \underline{T}_{T C P . i}\left(t_{n}\right)={ }^{T R} \underline{\underline{T}}_{I L, i} \cdot{ }^{I L} \underline{\underline{T}}_{T C P}\left(t_{n}\right)
$$

This TCP trajectory serves as an input for the kinematic simulation, see Fig 1 left, which needs to be executed for the evaluation process. The relevant component of the task intracorporeal suturing is identified to be stitching which results from the observations stated in Section 3.1. The suturing trajectory ${ }^{I L} \boldsymbol{T}_{T C P} \in R^{4 \times 4}$, see Equation (1), however contains the "stitching" and the "knot tying" motion. Aside from other factors, the influence of both trajectories, i.e. "stitching" and "stitching" + " knot tying" = "intracorporeal suturing”, will be presented in the next section. 


\section{Results}

In the following section, four different use cases will be presented. First of all, it is examined, whether a full "suturing" trajectory or only the "stitching" partition is used for the evaluation. It will be shown, that the application of only the "stitching" motion is sufficient. Afterwards, a comparison of different alternatives from the literature will be shown. The subsequent example contains a sampling over different RoM's for the roll axis of different alternatives to highlight its contribution to the SUR value i.e. how successful the task "stitching" is performed with respect to different orientations of the IL.

Example 1: The first example examines different task trajectories and their influence on the evaluation criteria SUR- and $d_{I B}$ value. The task trajectories in focus are 1) complete intracorporeal suture composed of "stitching" and "knot tying" and 2) the "stitching" trajectory solitary. The results of this comparison are presented in Table 1. As mentioned earlier, for the complete suture, the initial orientation of the "knot tying" motion is sampled as well, since the initial orientation of the "stitching" motion is sampled (see Section 3.2 Sampling of the line of incision). However, it is stated that the "knot tying" motion is independent of the line of incision and therefore needs not necessarily to be sampled. Thus, the results for the SUR values attained with the "suturing" motion illustrate a kind of worst case scenario. The results contain mean-values and standard deviation for the SUR value for different instrument designs from literature (number of joints, joint sequence and kinematic length are known from the respective publication) sampled over different $R o M s$ for the roll $\left( \pm 180^{\circ}- \pm 360^{\circ}\right)$ pitch and yaw $\left( \pm 70^{\circ}- \pm 100^{\circ}\right)$ axes. From Table 1, it can be observed that the "stitching" part of the suturing trajectory exhibits the main contribution to the evaluation results since the SUR values attained for the different reference trajectories "stitching" and "suturing" do not vary by more than the respective standard deviation. The decrease of the SUR values when performing a complete "suturing" motion can be addressed to the aforementioned assumed "worst case". In conclusion, the reference trajectory "stitching" was chosen for the following examples.

Table 1: Influence of the task trajectory on the SUR value from different instruments

\begin{tabular}{lcc}
\hline & $\begin{array}{c}\text { "stitching” + "knot tying” } \\
\text { mean(SUR) } \pm \text { std [\%] }\end{array}$ & $\begin{array}{c}\text { "stitching” } \\
\text { mesign }\end{array}$ \\
\hline Madhani [5] & $28.8 \pm 8.2$ & $36.2 \pm 6.5$ \\
Berkelmann [6] & $26.5 \pm 7.6$ & $33.1 \pm 6.9$ \\
Cooper [7] & $26.23 \pm 7.5$ & $36.4 \pm 4.5$ \\
Harada [8] & $29.7 \pm 8.4$ & $35.8 \pm 6.6$ \\
\hline
\end{tabular}



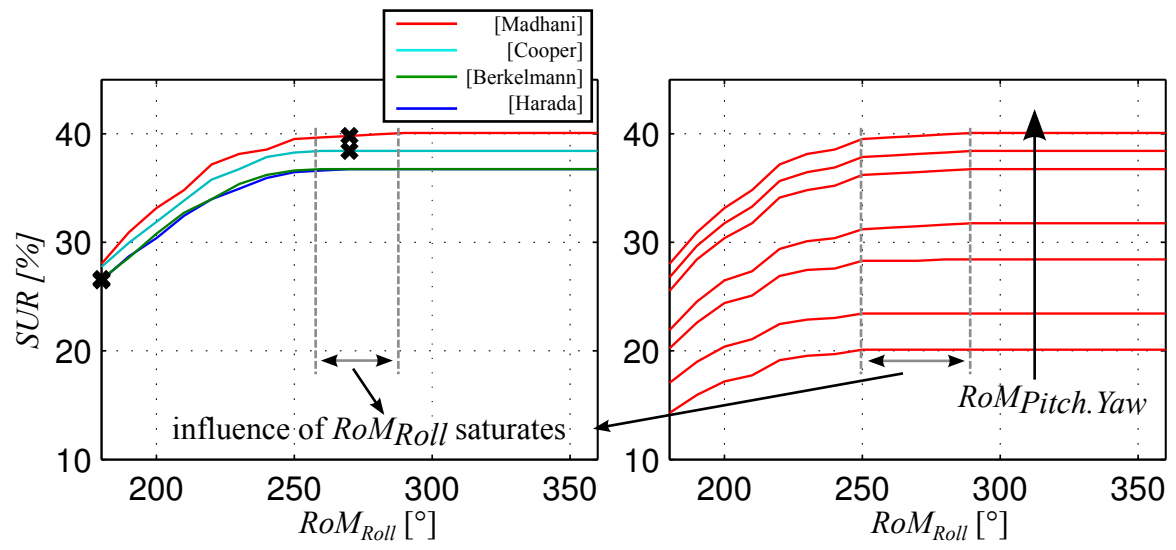

Fig 5: Influence of the RoM of the roll joint regarding different instrument designs (left) and varying RoM for the pitch and yaw axis of the design from Madhani [5] (right).

Example 2: In Table 2, a comparison of five instrument designs from the relevant literature is shown. As mentioned earlier, the reference trajectory is a "stitching" motion. By observation, these findings can be stated:

1. As kinematic length increases, the shaft distance criterion increases as well, e.g. $l_{k i n}=20 \mathrm{~mm}$ yields approx. $d_{I B}$ of $8 \mathrm{~mm}$ whereas $l_{k i n}=10 \mathrm{~mm}$ yields approx. $d_{I B}$ of $5 \mathrm{~mm}$

2. The low SUR $6.4 \%$ design [4] is due to the small range of the pitch and yaw joints with $\pm 45^{\circ}$ each, whereas the other alternatives realize $\pm 90^{\circ}$.

3. The superiority of designs [5], [7] are due to the high RoM in the roll axis of $\pm 270^{\circ}$, whereas the others only realize $\pm 180^{\circ}$.

Example 3: Point 3 from example 2 motivated us to further investigate the potential influence of the roll axis, especially if we consider the case of a multi turn roll axis, i.e. a roll axis without limits. Therefore, four designs with the same RoM for the pitch and yaw joints are considered [5]-[8] (see Fig 5, left). Furthermore, the design from Madhani [5] is investigated were the RoM of the pitch and yaw joints is varied from $\pm 70^{\circ}$ to $\pm 100^{\circ}$, (see Fig 5, right). By observation, we can conclude that the influence of the roll axis onto the SUR value saturates in between $\pm 255^{\circ}$ and $\pm 290^{\circ}$. This saturating characteristic is

1. independent of the evaluated instrument kinematic and

2. independent of the RoM of the pitch an yaw joints 
Table 2: Comparison of 5 instrument alternatives evaluated with the proposed method

\begin{tabular}{lcccccc}
\hline \multicolumn{1}{c}{ Design } & RoM $\left[{ }^{\circ}\right]$ & $\mathrm{N}_{\text {pitch,yaw }}$ & Type & $1_{\text {kin }}[\mathrm{mm}]$ & $\mathrm{SUR}[\%]$ & $\mathrm{d}_{\mathrm{IB}}[\mathrm{mm}]$ \\
\hline Madhani [5] & $\pm[270,90,90]$ & {$[1,1]$} & RPY & 10 & 39.5 & $5.0 \pm 0.8$ \\
Berkelmann [6] & $\pm[180,90,90]$ & {$[4,4]$} & RPYPYPYPY & 20 & 26.6 & $8.1 \pm 2.4$ \\
Seibold [4] & $\pm[180,45,45]$ & {$[1,1]$} & RPY & 30 & 6.4 & $15.0 \pm 0.5$ \\
Cooper [7] & $\pm[270,90,90]$ & {$[2,2]$} & RPYYP & 20 & 38.3 & $8.2 \pm 2.5$ \\
Harada [8] & $\pm[180,90,90]$ & {$[2,2]$} & RPYPY & 10 & 25.1 & $5.2 \pm 1.3$ \\
\hline
\end{tabular}

\section{Conclusion}

The paper at hand presented extensions and new results for the method published in [1] to evaluate alternative instrument kinematics w.r.t. their ability to perform a surgical task. The extensions included a more generalized representation of the reference task "intracorporeal suturing" by decomposition it into two subtasks, "stitching" and "knot tying". As "stitching" was identified to be the more complex motion and is also strongly dependent on the orientation of the incision line, it is the subtask representing the main challenge for an instrument and therefore it was proposed to be sufficient to use only this subtask in the evaluation method. The approach could be confirmed by the results presented in Section 4, example 1 .

The method incorporates criteria that reflect the performance of an instrument alternative with respect to the developed task and the consumed space while executing it. With these criteria, mechanical designers are able to compare new or known instrument kinematics quantitatively in order to develop them task specifically, see Section 4 example 2. Notably, the setup independence and the task generalization including specific motions that mainly affects the instruments capabilities contributes to a precise evaluation process and is the benefit of this method compared to others, e.g. [9], [10].

The method and its derived conclusions can be generalized to all instrument designs incorporating a roll axis as their first axis followed by an arbitrary sequence of pitch and yaw axis. This type of instrument is the most common design see e.g. [4]-[8]. To incorporate instruments with one or more intermediate roll axes, e.g. the instrument from [10], the instrument classification parameters as well as the kinematic simulation algorithm needs to be adapted which will be in focus of future work on this topic.

As conclusion we recommend for instrument designers to carefully design the range of motion of the roll axis, which should be $\pm 270^{\circ}$ for the tasks examined in this paper. As the performance saturates at $\pm 270^{\circ}$, the general assumption that a greater range of motion yields a higher performance could be reconsidered. 
Acknowledgments The authors would like to thank all surgeons for participating in the experiments, which are Prof. Rau, Dr. Authenrieth, Dr. Maurer, Dr. Do and PD Dr. Weikert.

\section{References}

[1]B. Deutschmann, R. Konietschke, and A. Albu-Schäffer, "Task-specific evaluation of kinematic designs for instruments in minimally invasive robotic surgery," in IEEE Conference on Intelligent Robots and Systems, 2013, pp. 3590-3597.

[2] a M. Derossis, G. M. Fried, M. Abrahamowicz, H. H. Sigman, J. S. Barkun, and J. L. Meakins, "Development of a model for training and evaluation of laparoscopic skills.," Am. J. Surg., vol. 175, no. 6, pp. 482-7, Jun. 1998.

[3] K. Moorthy, Y. Munz, A. Dosis, J. Hernandez, S. Martin, F. Bello, T. Rockall, and A. Darzi, "Dexterity enhancement with robotic surgery.," Surg. Endosc., vol. 18, no. 5, pp. 790-5, May 2004.

[4] U. S. Seibold, "An Advanced Force Feedback Tool Design for Minimally Invasive Robotic Surgery," TUM München, 2013.

[5] Madhani, "Articulated surgical instrument for performing minimally invasive surgery with enhanced dexterity and sensitivity," US5792135, 1998.

[6] P. Berkelman and J. Ma, "A Compact Modular Teleoperated Robotic System for Laparoscopic Surgery.,” Int. J. Rob. Res., vol. 28, no. 9, pp. 1198-1215, Sep. 2009.

[7] T. Cooper, "Surgical toll having positively postiionable tendon-actuated multi-disk wrist joint.pdf," US2003036748A1, 2003.

[8] K. Harada and K. Tsubouchi, "Micro manipulators for intrauterine fetal surgery in an open MRI,” IEEE Conf. Robot. Autom. 2005, no. April, pp. 2-7, 2005.

[9] M. C. Cavusoglu, I. Villanueva, and F. Tendick, "Workspace analysis of robotic manipulators for a teleoperated suturing task," in Conference on Intelligent Robots and Systems, 2001, vol. 4, no. Iros, pp. 2234-2239.

[10] D. Salle and P. Bidaud, "An Optimal Kinematic Design for a Highly Dextrous MIS Instrument," in On Advances in Robot Kinematics, Springer, 2004, pp. 399-408.

[11] T. Sheridan, "Evaluation of Tools and Tasks: Reflections on the Problem of Specifying Robot/Manipulator Performance," 1976, pp. 27-38.

[12] C. Cao, C. MacKenzie, and S. Payandeh, "Task and motion analyses in endoscopic surgery," 1996.

[13] C. Murakami and P. Odland, "Techniques Suturing Simple and Knot Tying," in Basic Surgical Concepts and Procedures, 1993, pp. 178-188.

[14] Chamberlain, "Suture Skills POD," 2014. [Online]. Available: http://www.thecgroup.com/product/robotic/skills/suture-skills-pod.

[15] H. Kang, "Robotic Knot Tying in Minimally Invasive Surgeries," in IEEE Conference on Intelligent Robots and Systems, 2002, no. October, pp. 1421-1426.

[16] A. Yershova, S. Jain, S. M. Lavalle, and J. C. Mitchell, "Generating Uniform Incremental Grids on SO(3) Using the Hopf Fibration.," Int. J. Rob. Res., vol. 29, no. 7, pp. 801-812, Jun. 2010.

[17] S. Jain, "Library for uniform deterministic se- quences/sets of samples over 2-sphere and $\mathrm{SO}(3), " 2009$. 